\title{
Numerical and Experimental Results of the Airflow in the Delft University KiteLab
}

\author{
Jeroen Breukels ${ }^{1}$ and Prof. Dr. Wubbo J. Ockels ${ }^{2}$ \\ Delft University of Technology
}

To be presented at the $37^{\text {th }}$ AIAA Fluid Dynamics Conference, Miami FL. 25 - 28 June 2007

\begin{abstract}
[Abstract] This paper presents the results of wind measurements taken in the ASSET KiteLab. KiteLab is a test facility on the roof of the faculty building where experimental kites can be flown and tested. It is a controlled environment where sensors and cameras collect data during flight test operations. The wind data has been analyzed and together with telltale tufts it has been used to validate a numerical model for the flow over the roof of the faculty building. This model was then used to study the flow in the KiteLab and identify a suitable test region in which kite tests can be conducted. What makes such a region suitable is a low wind path angle, allowing the kite to fly at an angle of attack which is close to its pitch angle.
\end{abstract}

\section{Introduction}

T $\mathrm{N}$ the past, kite design has been done mostly by empirical data and rules of thumb. The applications for which 1 kites were used did not demand scientific evaluation. Materials and prototyping can be done at such low cost that it does not warrant a scientific and systematic approach. Currently, the Faculty of Aerospace Engineering at the Delft University of Technology is conducting research into the application of kites for more industrial and largescale applications such as generation of energy and propulsion of large vessels. For these applications to operate at acceptable levels of efficiency, the system as a whole will have to be defined and evaluated through some sort of optimization procedure. These systems are simply too complex to be designed in a point-and-shoot manner. The kites themselves form an important part of the system and therefore, they will have to be evaluated and perfected through a scientific and meticulous process as well. Such an optimization process requires a scientific model of the kite. Using the old paradigm of rules of thumb is simply not sufficient for kites which are a part of a complex system. In order to develop a solid scientific model of a kite, a large number of tests have to be conducted in a controlled environment. Conducting flight tests in a wind tunnel is not practical because kites do not scale down to fit in a wind tunnel. Even in the biggest wind tunnel, the size of the measuring section puts severe limitations on the size of the kite and the cable length. Kite tests out in the field are a better option. However, it practice it is hard to create a controlled environment. Furthermore, the location of the Faculty of Aerospace Engineering would have it that large unobstructed pieces of terrain are far and few. In order to be able to do regular tests in a controlled environment, the plan for a kite laboratory on the roof of the faculty building was formalized.

\footnotetext{
${ }^{1} \mathrm{PhD}$ student, Faculty of Aerospace Engineering, Kluyverweg 1, 2629HS Delft, the Netherlands.

${ }^{2}$ Professor and Chair holder, Faculty of Aerospace Engineering, kluyverweg 1, 2629HS Delft, the Netherlands.
} 


\section{Laddermill}

The energy which is contained within the winds at high altitude (3000ft $-10000 \mathrm{ft}$ ) is tremendous. It is far greater than the wind at ground level which current windmill technology uses. This results in a higher dynamic pressure which allows lifting surfaces, with the same lift coefficient, to generate a much larger force. A force which can be used to generate electricity. In order to "harvest" this enormous energy resource, the laddermill uses large controllable kites to ascend and descend to drive a generator.

A regular kite will fly up without much effort. But when it has to come down, one has to pull on the tether to make it descent. For an aircraft it is the other way around. An aircraft requires big engines to climb, but it can glide down without spending fuel. Laddermill uses kites which combine the climbing capabilities of kites and the gliding capabilities of aircraft.

The principle of the Laddermill [Ref 12] is simple. A series of kites is connected to a long tether. The other end of this tether is wound on a drum connected to a generator. While the kites ascend from, for example, $3000 \mathrm{ft}$ to $10000 \mathrm{ft}$ altitude, they pull the tether off the drum, driving the generator and creating electrical energy. The lift is maximized by attitude and maneuvering. Once the kites reach their maximum altitude, their angle of attack is changed so that they generate very little lift. The tether is retrieved by rotating the drum. Once the kites reach their low altitude floor again, their angle of attack is increased and the process starts over. During retrieval of the cable, some energy will be spent. The difference in the energy created in the upward motion and the energy spent during the downward motion is the amount of energy created during one stroke. Figure 1 shows a schematic representation of the Laddermill.

\section{KiteLab}

A laddermill would not be possible without controllable kites, kites which can be flown as if they were aircraft. In order to design and build controllable kites, a kite testing facility was needed which would create a controlled environment within which safe and repeatable kite flight tests could be conducted. Due to the densely populated nature of the area around the Faculty of Aerospace Engineering, the idea was opted to build this facility on the roof of the faculty building.

KiteLab roughly consists of three separate parts. The monitoring section is where weather sensor data is collected and cameras are operated. This section is located on the floor directly below the roof surface (the $14^{\text {th }}$ floor). Also on this floor is the second section called the fabrication and preparation section. This is where kites are built and prepared for flight testing. The third section is located on the roof and is called the operations section. This is where the kites are flown. The operations section consists of two 25 meter high towers between which the flight tests are conducted. The towers have a number of functions. First and foremost, they secure the kite in case of loss of control. The kites are secured through a metal ring which is suspended between the towers. In case of a crash, this ensures that the kite does not

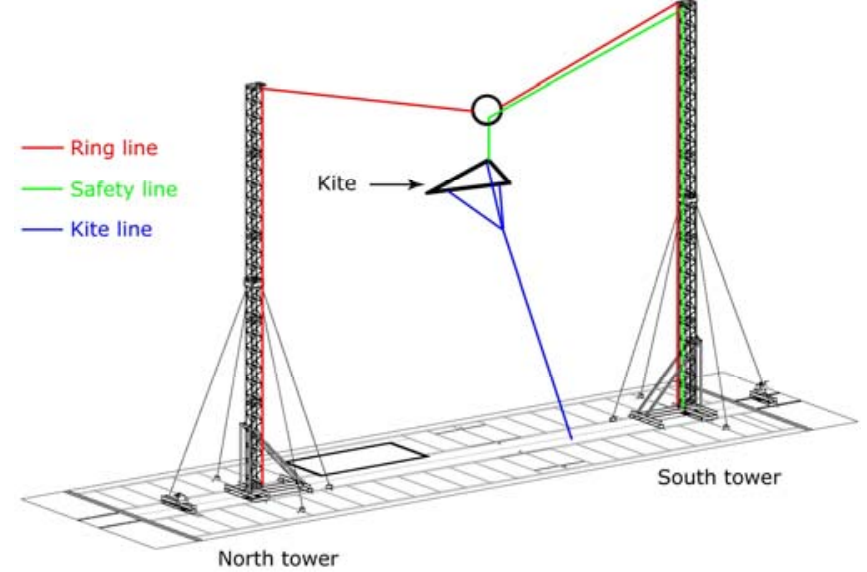

Figure 2. Rigging of kites in KiteLab. 
hit the building itself. Figure 2 shows the rigging concept for kite tests in the operations section.

Furthermore, the towers function as a platform for the sensory equipment such as wind sensors and camera's. The faculty building is 52.5 meters high which is considerably higher than any other building in close vicinity. This creates an uninterrupted airflow on the building. The presence of the building itself, however, does have a strong impact on the flow over the roof. The presence of wind sensors in the towers provide a unique opportunity to closely investigate the flow over the faculty building. In order to perform valid flight tests, a thorough knowledge of the flow over the building is required.

\section{Wind measurements}

The presence of the towers on the roof provides a good platform for a number of wind sensors. Wind sensors were placed in pairs at two different locations on the tower at the south end of the roof. One pair of sensors was placed at 12 meters above the roof surface, and another pair of sensors was placed in the top at 25 meters above the roof surface.

The sensors themselves are ultra-sonic wind sensors which have no moving parts. They measure the time of delay of sound waves moving within the sensor and by this time delay, they are able to determine wind speed and wind direction. They are 2 dimensional sensors, basically determining the velocity magnitude and the direction in one plane (generally a horizontal plane). The sensors have been calibrated in a wind-tunnel [Ref 3] and they were found to have a $2 \%$ maximum error in wind velocity magnitude and a 0.52 degrees maximum error in wind direction. The results of the wind tunnel tests are displayed in figure 3.
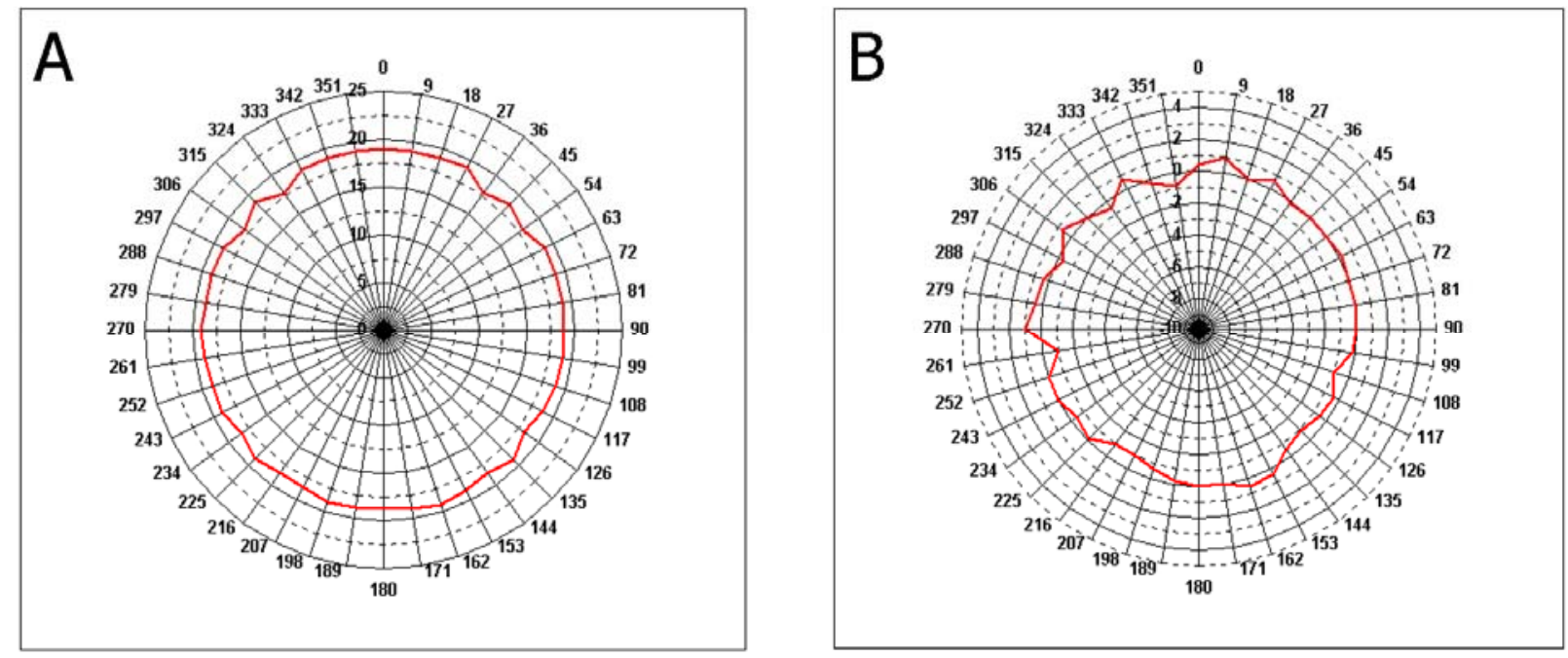

Figure 3. Results of the wind tunnel tests performed on the ultra-sonic wind sensor [REF 3].

What needs to be measured is a 3-dimensional wind vector. This is why two 2-dimensional wind sensors are required. By placing the wind sensors at an angle of 90 degrees with respect to each other, it is possible to measure the wind velocity magnitude, the wind direction and the path angle of the wind. The path angle of the wind is defined as the angle between the wind vector and the horizontal plane. Due to the presence of the building, it is expected that the wind is not perfectly horizontal. This path angle is essential in determining the angle of attack of a kite flying between the towers. Figure 4 shows the configuration of two wind sensors.

Due to the nature of the sensors the maximum "out-of-plane" angle in which they can measure the wind accurately enough is 15 degrees. This puts a limit on the number of measurements which can be used. If the wind blows directly on the side of the array, the top, straight-up sensor will be perfectly able to measure the speed and the direction, but the lower horizontal sensor will experience a wind directly on its top and

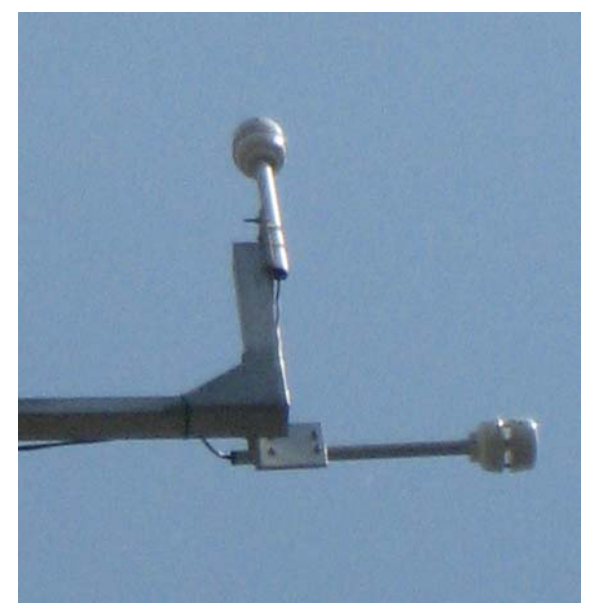

Figure 4. Wind sensor configuration 
will therefore not be able to do an accurate measurement. Therefore, only the measurements on the wind in a plus and minus 15-degree cone directly on the building can be taken into account.

The system logs data every second on a computer on the $14^{\text {th }}$ floor. The data was averaged in one hour blocks according to conventional wind measurement practice [REF 2]. The undisturbed wind velocity was measured at a local airfield close to the faculty building (Rotterdam airport). This data was used in combination with the wind velocities measured on the roof to determine the increase in wind velocity over the roof due to the presence of the building. In figure 5 the undisturbed wind velocity is plotted against the local wind velocity. The grey, upper line displays the velocities measured at 12 meter above the roof surface, half-way up the tower. The blue, lower line displays the velocity in the top of the tower, at 25 meters above the roof surface. The error bars indicate the standard deviation within the dataset. As can be seen, there is a significant velocity increase at 12 meters above the roof. The velocity increase at 25 meters above the roof is notably less. The increase in speed over the roof is the result of a venturi effect. The increase in local wind velocity relative to the undisturbed velocity is caused by an increased venturi effect due to the thickening of the turbulent region directly over the roof. This turbulent region is of great importance to the validity of kite tests to be performed in the KiteLab. To get a clearer picture of this turbulent region, the path angle of the wind was studied at different wind velocities. Figure 6 shows the path angle of the flow at both 12 meters (brown) and 25 meters (blue) above the roof surface as a function of the undisturbed flow velocity. As can be seen, increased wind velocity, the path angle increases as well. This effect is more pronounced for the position at 12 meters above the roof surface. This is the position where the local wind velocity is high and where there is a high wind path angle. This location would therefore be unsuitable to perform kite tests. The increased angle of attack of the wind would create problems. The kite would fly at a very low or even negative pitch angle. If the kite now moves into an area which has a much lower wind path angle, or a situation occurs where the wind path angle is suddenly decreased, the kite would enter a stall. Many kites are constructed as such that in case of a front stall, they collapse. At which point the test would have to be ended and the kite would have to be re-launched. It can also be seen from the error bars that the wind is gustier at 12 meters above the roof, further complicating the flight test process. At 25 meters over the roof surface, the wind path angle is significantly reduced. Here, the error bars are also narrower, indicating a more stable airflow.

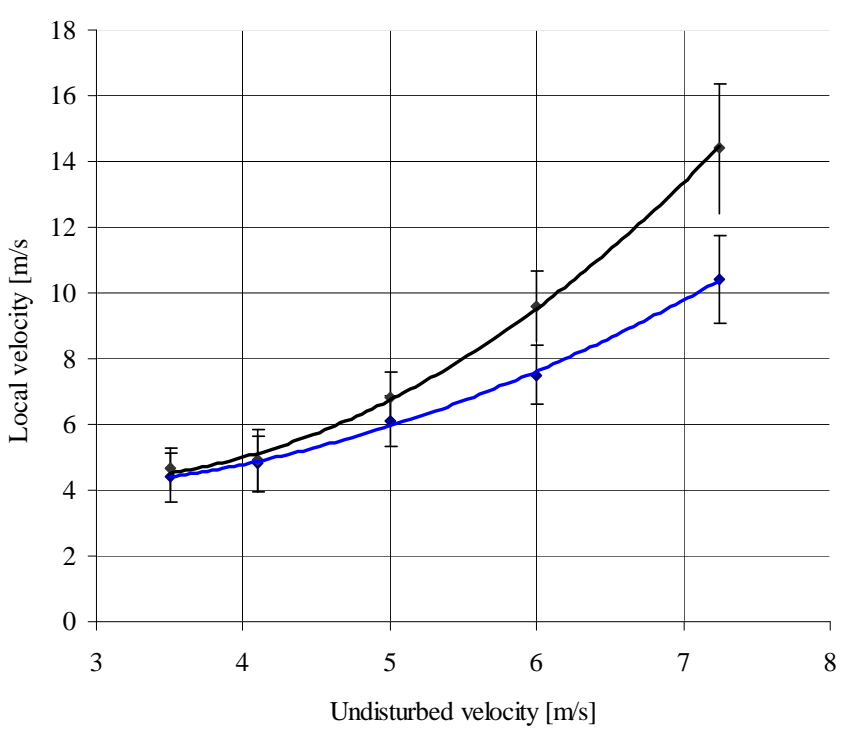

Figure 5. The local wind velocities plotted against the undisturbed wind velocities. Blue indicates the top of the tower, and grey indicates the middle of the tower.

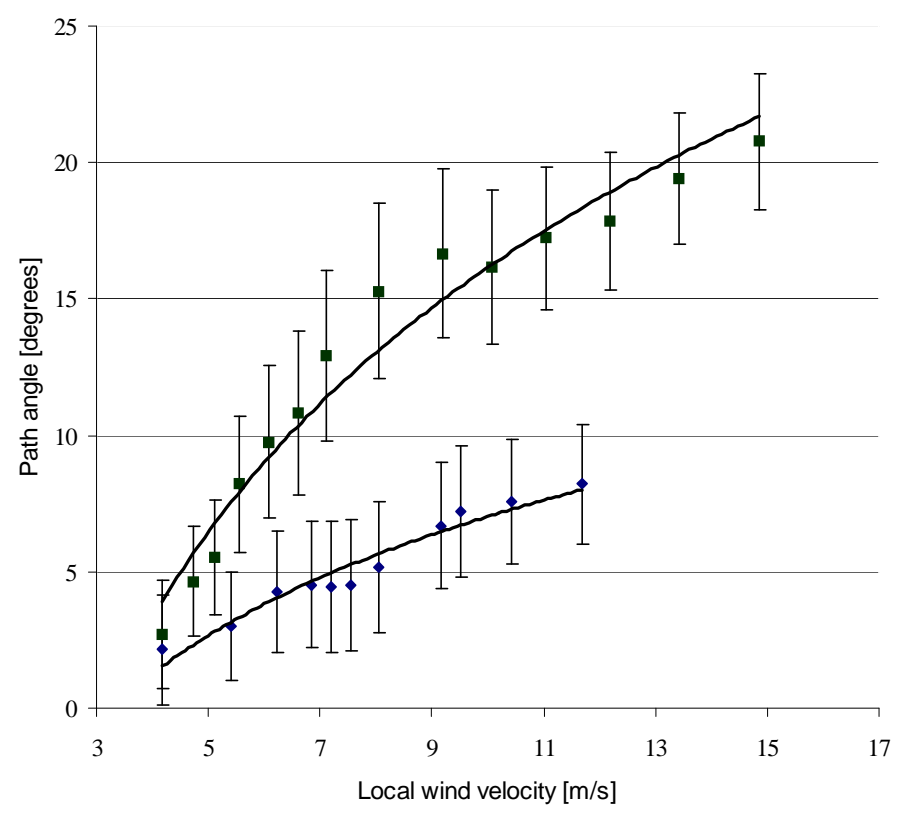

Figure 6. The wind path angle plotted against the local wind velocities. Blue indicates the top of the tower, and brown indicates the middle of the tower.

The measurements in figures 5 and 6 were all taken using the wind sensors which are attached to the outside of the south tower. The actual test section where kites are flown are much more in the middle of the building. Even though the measurements using the wind sensors give very viable data, it is also required to observe the flow in the 
middle between the towers. During regular flight operations, the test section has to remain uncluttered to prevent entanglement. It is therefore important that a link is made between the measurements done on the side of the tower and the actual wind velocity vector in the test section. In order to visualize the vortex which exists close to the roof surface, three very thin tethers were suspended in between the towers from the roof surface to an altitude of 25 meters above the roof surface. The tethers were spaced 5 meters apart. From these very thin tethers, small woolen tufts were hung at every 0.5 meter in altitude. Figure 7 shows this setup. The tufts were observed at different wind speeds. Determining an exact angle from these tufts is extremely difficult to do. What could be observed very clearly though was that the tufts close to the roof were moving in a very erratic way while the tufts towards the top were almost stationary. The border between the erratic moving tufts and the stationary tufts was clearly visible. The erratic moving tufts were in very instable air. It is here where the influence of the building itself is so great, that it disturbs the flow and creates a turbulent region. In this region, flight tests are impossible. But moreover, for flight tests to be conducted well above this turbulent region, the kite would first have to be hoisted through this unstable flow. This can potentially cause problems due to the fact that some kites are fragile and do not cope well with radically changing wind speeds and directions. The size or thickness of this unstable region differs with wind velocity. Figure 8 shows the thickness of the unstable region as a function of the wind speed. This wind speed is

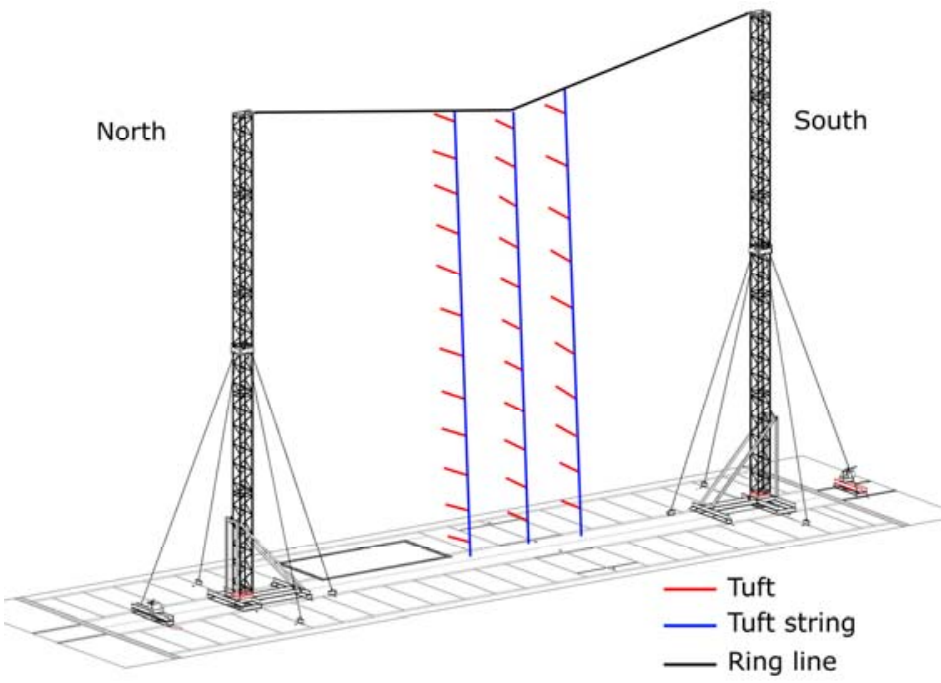

Figure 7. The woolen tufts installed between the towers.

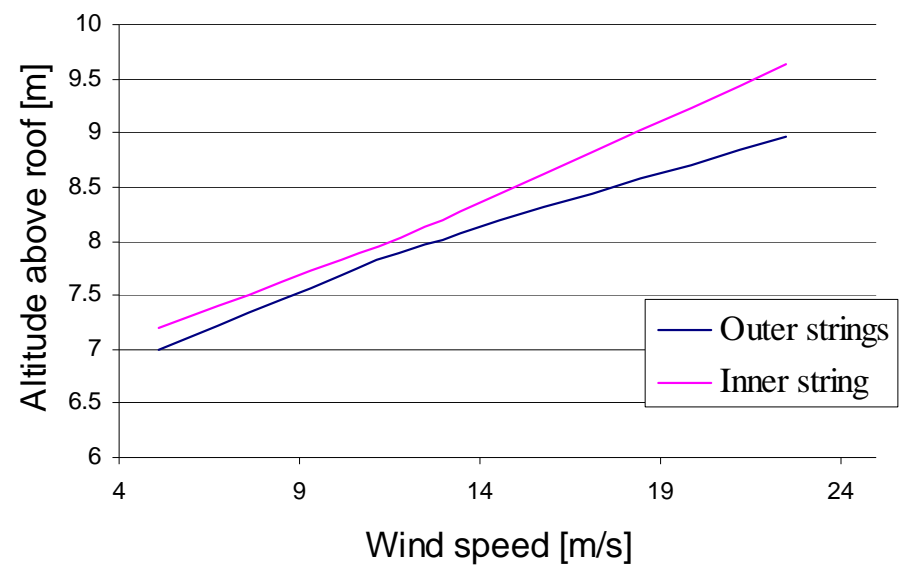

Figure 8. Thickness of the turbulent region as a function of local wind speed. measured at the top of the south tower and is therefore a locally measured value. The values of the outer strings were virtually identical due to symmetry. The measurements were only done with a wind direction which was straight onto the building. As can be seen in figure 8 , the thickness of the turbulent region can be as much as 9.5 meters in high wind velocities. Generally, kite tests would be done in wind speeds of around 10 meters per second (local value). This would result in a turbulent layer of close to 8 meters thick. That leaves a good 17 meters above this layer where the air is stable enough for kite testing.

\section{Numerical model}

From the tufts and the wind measurements it became apparent that there is a turbulent region directly above the roof surface and that the path angle of the wind is effected by the presence of the building itself. Even though the tufts provided information on the thickness of the turbulent region, it provided no information on specific wind velocities and path angles in the test section itself. Therefore, a numerical CFD model was made of the building. This model equals a block of space, 200 meters long, 150 meters wide and 125 meters high. In the middle, the building is represented by a block. On top of this block, the towers are modeled as slender columns. The roof itself is modeled including the 1 meter high railing all around the roof surface. The entire model uses 13 million cells. At one side of the model, a velocity inlet was created which provided a wind velocity of $7.2 \mathrm{~m} / \mathrm{s}$. on the opposite side, a pressure outlet enables the air to exit the model. The sides of the model are also transparent and can let air in or out. 
The ground and the surface of the building and towers are modeled as walls. The model was meshed using an unstructured mesh, after which it was processed using Fluent. The model needs to be checked against the measured values. Table 1 shows the measured values of local wind velocity and the values obtained from the numerical model. As can be seen, the numerical model slightly underestimates the local wind speed values. But taken into account that the spread of the measured values is quite large (as can be seen by the relatively wide error bars in figure 5) these deviations fall well within the accuracy of the measured values.

For the wind path angle we can do the same comparison. The path angle of the wind was measured and depicted in figure 6 . Table 2 gives a comparison between the measured values and the modeled values. Again, the model underestimates the values. And again, the deviations fall well within the error bars depicted in figure 6 .

The next step is to compare the thickness of the turbulent region between the towers. As was seen in figure 8, the thickness of the turbulent region was 7.8 meters at a local wind speed of approximately 10 meters per second. This 10 meters per second as measured in the top of the tower is comparable to the local wind speed in the numerical model, which is $9.78 \mathrm{~m} / \mathrm{s}$ (table 1). Figure 9 shows the dynamic pressure in between the towers. In a region of high turbulence, there is a low dynamic pressure. As can be seen in figure 9 , this low dynamic pressure is indicated in blue. The red-white bar along the tower indicates the altitude over the roof. Every block is equal to one meter altitude. In can be seen that the thickness of the modeled low dynamic pressure region is very close to the measured value of 7.8 meters. Together with the comparison values in

\begin{tabular}{|c|c|c|}
\hline position & Measured value & Modeled value \\
\hline 12 meters & $14.42 \mathrm{~m} / \mathrm{s}$ & $13.86 \mathrm{~m} / \mathrm{s}$ \\
\hline 25 meters & $10.44 \mathrm{~m} / \mathrm{s}$ & $9.78 \mathrm{~m} / \mathrm{s}$ \\
\hline
\end{tabular}

Table 1. Comparison of local wind speeds obtained from measurements and numerical modeling

\begin{tabular}{|c|c|c|}
\hline position & Measured value & Modeled value \\
\hline 12 meters & $12^{\circ}$ & $9.72^{\circ}$ \\
\hline 25 meters & $5^{\circ}$ & $4.74^{\circ}$ \\
\hline
\end{tabular}

Table 2. Comparison of the wind path angle obtained from measurements and numerical modeling

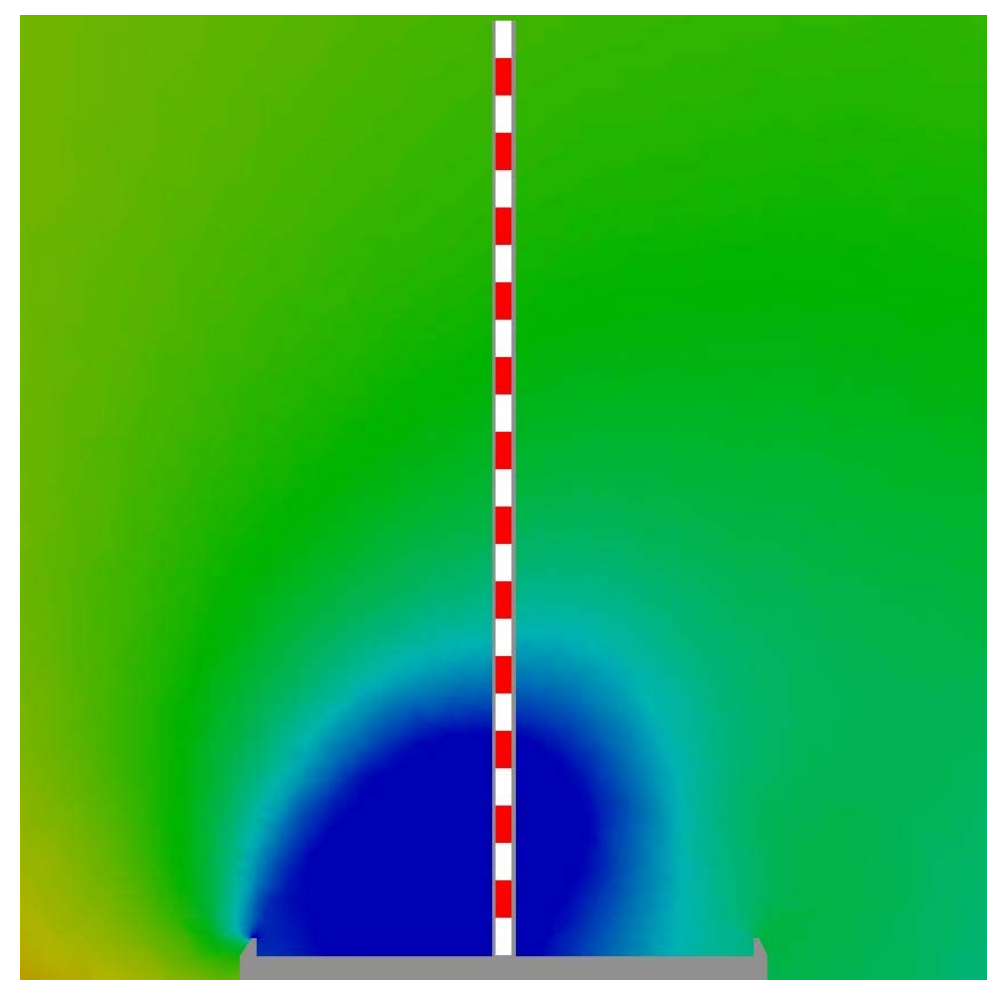

Figure 9. Modeled representation of the turbulent region.

tables 1 and 2, this leads to believe that the model indeed accurately reflects the actual situation in the KiteLab.

As was both measured and modeled, the path angle of the wind between the towers is not insignificant. We can employ the model to plot the path angle of the wind at different altitudes over the roof surface. In order for kite tests to be successful, one would ideally look for a region where the wind is perfectly horizontal. It is possible to fly a kite a maximum altitude of twice the height of the towers. In this situation, when a failure occurs, the ring line (see figure 2) is still capable of securing the kite and keeping it from hitting the side of the building. The question is wetter or not twice the altitude of the building is sufficient altitude to reach a region with a very low wind path angle. If this region exists, it would be ideally suited to conduct flight tests. As altitude above the towers increases, flight tests become more difficult to conduct. Testing in the region close to twice the altitude of the towers is theoretically possible, but a lower altitude would be preferred, if possible. At higher altitudes, observations are harder to make, and the amount of influence on the flight path by using the lines on the towers significantly decreases. Furthermore, in the event of a loss of control, the resulting crash will be more severe as altitude above the towers increases. 
Figure 10 shows the path angle between the towers. These values are obtained from the numerical model. As can be seen, the path angle remains significant for large altitudes over the roof surface. They are only dropping to values close to 3 degrees at altitudes of 40 meters above the roof surface. In between the top of the towers, the wind path angle is still a good 6 degrees. And although this path angle can be taken into account during flight tests, it is far more desirable to have a lower wind path angle. One also has to consider that for higher wind velocities, the wind path angle will increase even more. In regions with a lower wind path angle, this effect would be reduced as well. In order to locate a region with a lower wind path angle, streamlines were drawn using the numerical model. Figure 11 shows the resulting stream lines. As can be seen, in the region above and behind the building there exists an area with a wind path angle of close to zero. Directly above the tower we see the familiar picture of a slowly decreasing wind path angle, as was plotted in figure 10 . But about 10 meters behind this area, the path angle becomes zero. The fact that this region is not directly over the roof surface is of no direct consequence. The safety lines are able to secure a kite in the event of a failure just as well in this region as in the region directly over the surface of the roof. Moreover, a test region slightly downwind will also create more space on the roof to place the ground station. Since the cable to a kite is never straight up, this slight downwind position will ensure that the ground station does not have to be placed on the very edge of the building.

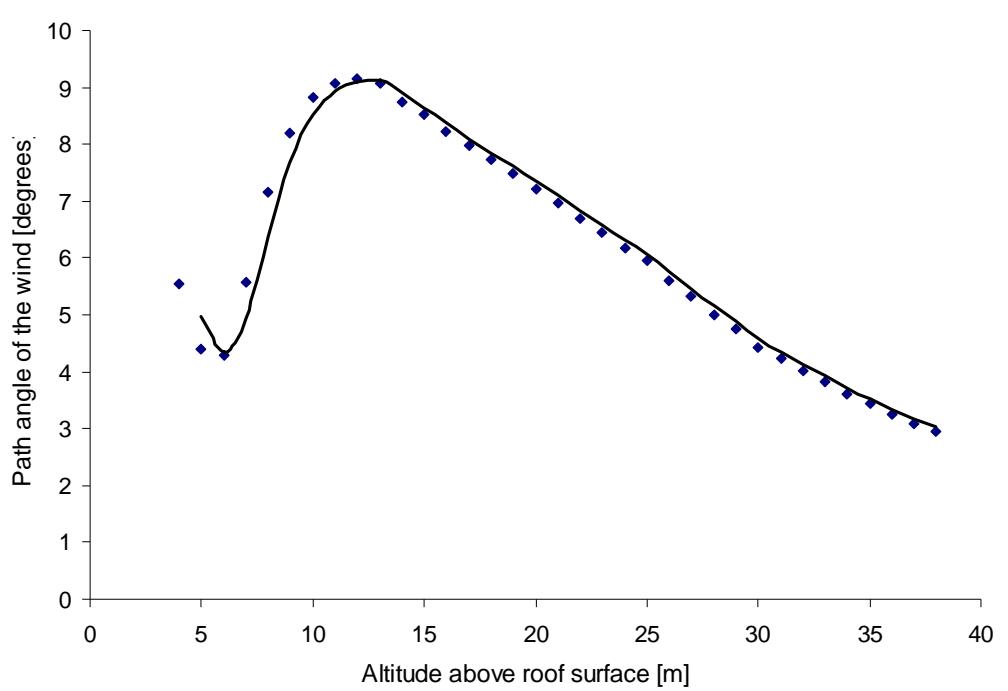

Figure 10. The wind path angle between the towers as a function of altitude over the roof surface.

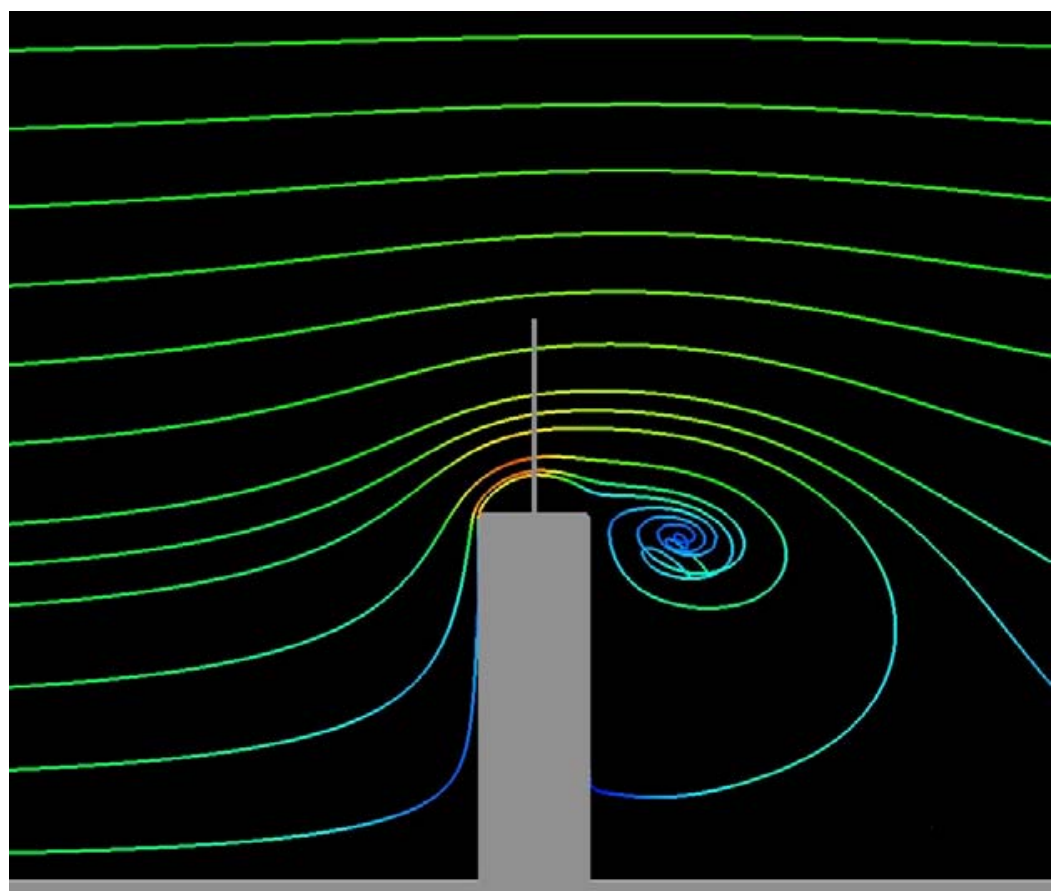

Figure 11. Streamlines over the roof between the towers.

\section{Conclusions and recommendations}

In this paper, it was shown that a numerical model can be a valuable tool during flight test operations in the KiteLab facility. Measurements taken indicate that the wind close to the roof surface is quite gusty and unstable. At higher altitudes, the airflow settles down significantly. The air here is stable enough for flight tests to be performed. The wind path angle is greatly influenced by the presence of the building. Both the numerical model and the wind measurements agree. The wind path angle at the top of the towers is typically in the range of 5 to 10 degrees. A region with a path angle close to zero degrees exists behind and above the towers. It is recommended to perform 
more numerical simulations at different wind velocities to further investigate the relationship between measurements and numerical data.

\section{Acknowledgments}

This work is part of the Laddermill project which is sponsored by the Delft University of Technology, the port of Rotterdam, the University of Groningen, the town of Delft, Gasunie, Fugro, Shell Research, Prolyte products, energy valley, the Town and Province of Groningen.

\section{References}

1. Breukels, J., 2006, "KiteLab” ASSET Internal report. Faculty of Aerospace Engineering, Delft University of Technology

2. DeFelice, T.P., "An introduction to meteorological instrumentation and measurement" Prentice Hall, Upper Saddle River, New Jersey 07458, ISBN 0-13-243270-6.

3. Free Technics B.V. "Etalonnade du CV3F”, Internal report.

4. Breukels, J., 2005, "KitEye WP3 - WP8” ASSET Internal report. Faculty of Aerospace Engineering, Delft University of Technology

5. Veldman, S.L., Vermeeren, C.A.J.R., Bersee, H.E.N., Bergsma, O.K., Conceptual design of a high altitude kite, Faculty of Aerospace Engineering, Delft University of Technology, AIAA-2002-1735.

6. Bryant L.W., Brown W.S., Sweeting, N.E., Collected researches on the stability of kites and towed gliders, Aeronautical Research Council R\&M 2303.

7. Breukels J., Design of a high altitude inflatable kite, Delft University of Technology, Faculty of Aerospace Engineering, August 2003.

8. Voortgangsverslag van het Stratow-projekt, ir. J Melkert, 1992. Delft University of technology, Faculty of Aerospace Engineering

9. John D. Anderson, Fundamentals of Aerodynamics second edition, McGraw-Hill 1991

10. Dictaat D-3-2 Flight Dynamics, November 1999, Delft University of Technology, Faculty of Aerospace Engineering

11. Dickinson, B. Aircraft stability and control for pilots and engineers, sir Isaac Pitman \& Sons, London 1968.

12. Ockels, W.J., "Laddermill, a novelconcept to exploit the energy in the airspace” Aircraft Design 4 (2001) p81p97. 